\title{
A UTILIZAÇÃO DO SOFTWARE GEOGEBRA COMO RECURSO METODOLÓGICO PARA O ENSINO DE FUNÇÃO POLINOMIAL DO PRIMEIRO GRAU NO 9 ANO
}

\author{
Fernando Temporini Frederico ${ }^{1}$, Aline Alves de Oliveira ${ }^{2}$, Débora Cristina Curto da Costa Bocato ${ }^{3}$, \\ Geralda de Fátima Neri Santana ${ }^{3}$, Weslley Cabral de Oliveira ${ }^{2}$ \\ Universidade Estadual de Maringá - UEM. ${ }^{1}$ Pós-graduação em Educação para a Ciência e a Matemática, Maringá - PR. \\ ${ }^{2}$ Mestrado, bolsista CAPES/Fundação Araucária, Maringá - PR. ${ }^{3}$ Mestrado/PCM, Maringá - PR. E-mail: \\ ftfrederico@gmail.com
}

\section{RESUMO}

Este estudo pretendeu investigar, qualitativamente, como o software Geogebra pode contribuir para processos que envolvem o ensino e a aprendizagem, na disciplina de Matemática, do conteúdo de Função Polinomial do Primeiro Grau com alunos do 9o ano do ensino fundamentalem uma escola pública no município de Maringá, Brasil. Para isso, desenvolveram-se atividades de ensino em dois momentos: primeiramente em sala de aula de maneira convencional, sem o uso de recursos tecnológicos e, em seguida, no laboratório de informática utilizando o software Geogebra. Ao final da pesquisa aplicou-se questionário semiestruturado, onde constatou-se que o uso do Geogebra dinamizou, potencializou e tornou mais significativa a aprendizagem dos alunos. Desta forma, espera-se que esses resultados suscitem discussões e reflexões frente a inserção das TIC no ambiente educacional em busca de uma educação com mais qualidade.

Palavras-chave: Ensino; Aprendizagem; Função Polinomial do Primeiro Grau; Software Geogebra; TIC.

\section{THE SOFTWARE GEOGEBRA UTILIZATION AS A METHODOLOGICAL RESOURCE FOR THE TEACHING OF THE POLYNOMIAL FUNCTION OF FIRST DEGREE IN $9^{\text {TH }}$}

\begin{abstract}
This study intended to investigate qualitatively how the software Geogebra can contribute to the processes that involve teaching and learning in the discipline of mathematics, the content of Polynomial Function of First Degree with students from 9th grade of basic education in a public school in the city Maringa, Brazil. To do this, educational activities were developed in two stages: first in class in conventional manner, without the use of technological resources and then in the computer lab using Geogebra software. At the end of the research was applied semi-structured questionnaire, where it was found that the use of Geogebra streamlined, potentiates and made most significant student learning. Thus, it is expected that these results rouse discussions and reflections across the insertion of ICT on education environment in search of a better quality education.
\end{abstract}

Keywords: Education; Learning; Polynomial Function of the First Degree; Geogebra software; ICT. 


\section{INTRODUÇÃO}

É cada vez mais fácil percebermos em vários seguimentos da sociedade, inclusive na escola, um número crescente de adeptos ao uso de várias tecnologias da informação e comunicação. Aparelhos como notebooks, tablets, smartphones e telefones celulares são cada vez mais comuns nas salas de aula, mesmo que, ainda, não utilizados para a aprendizagem. Em sua maioria, nossos alunos pertencem a uma nova geração, segundo Toledo, Albuquerque, Magalhães (2012), são aqueles nascidos entre 1995 e 2010, a chamada geração Z, segundo Prensky (2001), nativos digitais. Esses alunos estão intensamente conectados e disponíveis através de seus dispositivos móveis em um novo tipo de grupo social, os virtuais.

Deste modo, as $\mathrm{TIC}^{1}$ correspondem a todas as tecnologias que interferem na vida dos seres. O uso dessas ferramentas vem sendo debatido de forma intensa em todos os segmentos do conhecimento humano, como na indústria, comércio, no setor de investimentos e na educação, sendo representada pelos novos meios de ensino, a fim de potencializar o processo de aprendizagem presencial e principalmente à distância.

Este debate se deve principalmenteao aparente desinteresse que boa parte de nossos alunos tem demonstrado diante de nossas aulas e que, geralmente, possuem fortes características da educação tradicional, modelo educacional pertencente às gerações anteriores a qual pertencemos, como a $\mathrm{X}$ e $\mathrm{Y}$ e que atualmente, não atendem as expectativas da maioria dos alunos. Outro fator preponderante está relacionado a linguagem, como afirma PRENSKY (2001):

[...] the single biggest problem facing education today is that our Digital Immigrant instructors, who speak an outdated language (that of the pre-digital age), are struggling to teach a population that speaks an entirely new language. This is obvious to the Digital Natives - school often feels pretty much as if we've brought in a population of heavily accented, unintelligible foreigners to lecture them. They often can't understand what the Immigrants are saying. What does "dial" a numbermean, anyway? (PRENSKY, 2001, p. 2).

Devido a esse impasse, recaímos em um dilema profundamente debatido e conhecido por todos que estão diretamente envolvidos ao âmbito educacional: a formação inicial, continuada e a prática pedagógica dos profissionais da educação. Diante dessa disparidade entre as gerações, o professor de hoje deve se adequar as novas tendências tecnológicas, uma vez que sua formação inicial pode não ter privilegiado o uso de tais recursos e que a realidade educacional atual anseia por profissionais mais flexíveis, reflexivos e que busquem abordagens metodológicas mais dinâmicas para com o processo de ensino e aprendizagem.

\footnotetext{
${ }^{1}$ TIC: Tecnologias da Informação e Comunicação.
} 
Considerando tais diferenças entre as várias gerações, $X, Y, Z$, é possível de se concordar que seria necessária uma mudança radical em nossa prática pedagógica? Se introduzirmos em nosso cotidiano profissional metodologias que envolvam as TIC, os resultados seriam diferentes daqueles vivenciados ultimamente? Ou ainda, com as TIC, a aprendizagem passaria a ser mais importante e efetiva aos olhos de nossos alunos?

A sociedade com o passar do tempo transformou-se muito, o que não ocorreu na mesma proporção no ambiente escolar, uma vez que com os inúmeros avanços tecnológicos que vieram para facilitar a vida humana, a escola pouco os agregou em sua rotina cotidiana. Além da própria internet, do computador, dos games, smartphones e tablets, dos inúmeros softwares desenvolvidos para diversos fins e das incontáveis aplicações ligadas à informática e sua engenharia, todas podem ser consideradas ferramentas essenciais que nos auxiliam diariamente. Desta forma é impossível não considerá-las como relevantes para fins educacionais, uma vez que nossos adolescentes, em sua grande maioria, são usuários frequentes de tais ferramentas e de outros recursos que denominamos por TIC. Quanto mais mergulhamos na sociedade da informação, mais rápidas são as demandas por respostas instantâneas. As pessoas, principalmente as crianças e os jovens, não apreciam a demora, querem resultados imediatos. Adoram as pesquisas síncronas, as que acontecem em tempo real e que oferecem respostas quase instantâneas (MORAN, 2000, p. 20).

Dentre os recursos tecnológicos citados anteriormente, daremos ênfase aos softwares, pois acreditamos que tal ferramenta potencializa a aprendizagem dos alunos ao decorrer do processo de ensino.

Os softwares são programas que podem ser programados para inúmeros fins, sendo empregados nos mais variados setores da sociedade e, no meio educacional, pode estar presente em todos os níveis e modalidades de ensino. Eivazian (2008) esclarece que o software oferece aos alunos uma oportunidade de serem agentes da sua própria aprendizagem, o que lhes possibilita tomar decisões e analisarem dados seguindo passos relacionados ao método científico, sem, entretanto, estarem presos ao rigor do mesmo, sendo um recurso que pode contribuir para a formação do raciocínio crítico do aluno, contribuindo para que seja mais participativos na sociedade a qual pertence. A Matemática, assim como outras disciplinas, como Física e Química, por exemplo, são áreas do conhecimento mencionadas, por muitos alunos, como aquelas que demandam de muita abstração, principalmente, quando consideramos a vertente Matemática. Sendo assim, qualquer recurso que possa contribuir para a superação destas dificuldades deve ser considerado, como é o caso do software Geogebra. 
O software Geogebra foi criado por Markus Hohenwarter, o projeto foi iniciado em 2001, na Universität Salzburg, e tem prosseguido em desenvolvimento na Florida Atlantic University. É um software gratuito de Matemática dinâmica que foi desenvolvido para ser utilizado em ambiente de sala de aula. Reúne recursos de geometria, álgebra e cálculo. Ele possui todas as ferramentas tradicionais de um software de geometria dinâmica: pontos, segmentos, retas e seções cônicas. E nele encontramos, também, equações e coordenadas que podem ser inseridas diretamente.

O software Geogebra traz muitas vantagens para atividades desenvolvidas no ensino da Matemática se comparadas ao trabalho no papel ou no quadro, como movimentar as figuras em diversas direções, comparar e voltar ao aspecto inicial. Além disso, não serve apenas para trabalhar com mais agilidade e buscar diversos caminhos de resolução de problemas, mas também para checar se o que foi feito está correto.

Com o uso do software de geometria dinâmica Geogebra, foi implementado uma sequência didática, a fim de avaliar as contribuições da inserção desse software na disciplina de Matemática, durante o processo de ensino e aprendizagem dos conceitos inerentes ao estudo do conteúdo Função Polinomial do Primeiro Grau em uma turma de alunos do 9o ano do Ensino Fundamental de uma escola pública da rede estadual de ensino, pertencente ao município de Maringá, Paraná, Brasil.

\section{PROCEDIMENTOS METODOLÓGICOS}

Inicialmente, foi realizado levantamento exploratório da literatura, abrangendo discussões sobre o uso das TIC no ensino da Matemática, especialmente no que diz respeito à aplicação do software Geogebra.

Paralelamente, foi realizada a pesquisa ${ }^{2}$ de campo com 20 (vinte) alunos do 9 ano do Ensino Fundamental no Colégio Estadual Ipiranga, no início do 2o bimestre de 2014. A presente pesquisa é de caráter qualitativo. Para Creswell (2007), a pesquisa qualitativa é fundamentalmente interpretativa. Isso significa que o pesquisador interpreta os dados coletados. Desse modo, "[...] o objetivo principal do investigador qualitativo é o de construir conhecimento" e não apenas opinar (BOGDAN E BIKLEN, 1999, p. 67). Assim, o interesse esteve centrado nas possíveis contribuições do software Geogebra para a aprendizagem do conteúdo Função Polinomial do Primeiro Grau de modo dinâmico e interativo.

\footnotetext{
${ }^{2}$ Pesquisa submetida ao COPEP - Comitê Permanente de Ética em Pesquisas com Seres Humanos e aprovada sob número 03014912.3.0000.010. 
Esse conceito foi abordado por uma sequência de atividades com 12 (doze) exercícios sendo trabalhados no decorrer do processo de ensino aprendizagem. Para tanto, a pesquisa foi desenvolvida em dois momentos distintos: primeiramente em sala de aula de maneira convencional utilizando material manipulável (papel milimétrico, régua, lápis, borracha), a fim de buscar a interpretação algébrica, que leva a formação da lei de formação de uma função. Após, os alunos foram levados ao laboratório de informática, onde tiveram o primeiro contato com o software Geogebra. Foi solicitado aos mesmos que começassem a aplicar no software as atividades desenvolvidas em sala. Para isso, sentaram-se em duplas para desenvolverem a sequência de atividades.

Ao final da pesquisa, foi aplicado um questionário semiestruturado composto por questões discursivas e objetivas visando investigar o ponto de vista dos alunos após o uso do recurso tecnológico de geometria dinâmica, Geogebra. Tais questões serviram de parâmetros para o objeto de estudo, garantindo deste modo, a uniformidade do entendimento dos alunos. Na sequência, passaremos aos resultados e discussões.

\section{RESULTADOS}

Após a intervenção pedagógica descrita anteriormente, o questionado aplicado foi constituído por 4 (quatro) questões. Os resultados apontaram que a utilização do referido software pode contribuir potencialmente para dinamização e abordagem do conteúdo matemático proposto: Função Polinomial do Primeiro Grau. Neste sentido, apresentamos na sequência a discussões acerca dos resultados obtidos.

\section{DISCUSSÕES}

As duas primeiras questões buscaram investigar sobre as possíveis dificuldades que os sujeitos envolvidos tiveram ao manusear o software.

As respostas apontaram que $75 \%$ dos alunos afirmaram não ter tido dificuldades em manusear o software durante a atividade proposta. Além disso, $60 \%$ dos sujeitos ainda argumentaram que este tipo de recurso deveria ser utilizado com mais frequência em outros conteúdos ligados à disciplina de matemática.

Diante dos apontamentos, verifica-se a expressiva contribuição que este tipo de ferramenta é capaz de proporcionar quando empregada de forma planejada e sistematizada que, neste caso, foi voltada ao ensino. Os softwares, assim como outras TIC são recursos que tem transformado nossa sociedade. 
Coll e Monereo (2010, p. 17) salientam que dentre todas as tecnologias criadas pela humanidade, aquelas que são capazes de representar e transmitir informação, ou seja, as TIC, são revestidas de uma importância especial, pois, são capazes de afetar praticamente toda a atividade humana, "desde as formas e práticas de organização social até o modo de compreender o mundo, de organizar essa compreensão e de transmiti-la para outras pessoas".

O computador, com certeza, é uma das ferramentas que tem contribuído para a transformação social e, também, da compreensão do mundo. Entretanto, houve de certa forma, há algum tempo atrás, o receio de que a máquina (computador) pudesse substituir o ser humano - o professor. Mas, percebeu-se que o papel do professor é insubstituível e, que o computador, seria mais um recurso que poderia ser utilizado para ajudá-lo e, não-, substituí-lo.

Sobre esta questão, Tajra (1998), afirma que:

No início da introdução dos recursos tecnológicos de comunicação na área educacional, houve uma tendência a imaginar que os instrumentos iriam solucionar os problemas educacionais, podendo chegar, inclusive, a substituir os próprios professores. com o passar do tempo, não foi isso que se percebeu, mas a possibilidade de utilizar esses instrumentos para sistematizar os processos e a organização educacional (TAJRA, 1998, p. 23).

Percebe-se, portanto, que tecnologias como o computador vêm se configurando como uma ferramenta capaz de contribuir efetivamente para processos que envolvem o ensino e aprendizagem.

A terceira questão objetivou identificar as possíveis contribuições proporcionadas pelo software: "Quais as contribuições que o software Geogebra Ihe proporcionou para que o conteúdo, função polinomial do primeiro grau, se tornasse significativo durante as aulas de matemática?" Os depoimentos dos alunos podem ser expressos em 4 (quatro) categorias: A: melhor compreensão da Função Polinomial do Primeiro Grau; B: melhor compreensão de elementos geométricos; C: melhor compreensão de gráficos e D: facilita e estimula a aprendizagem. Diante das respostas, apresentamos o gráfico 1: 
Gráfico 1. Contribuições indicadas pelos alunos

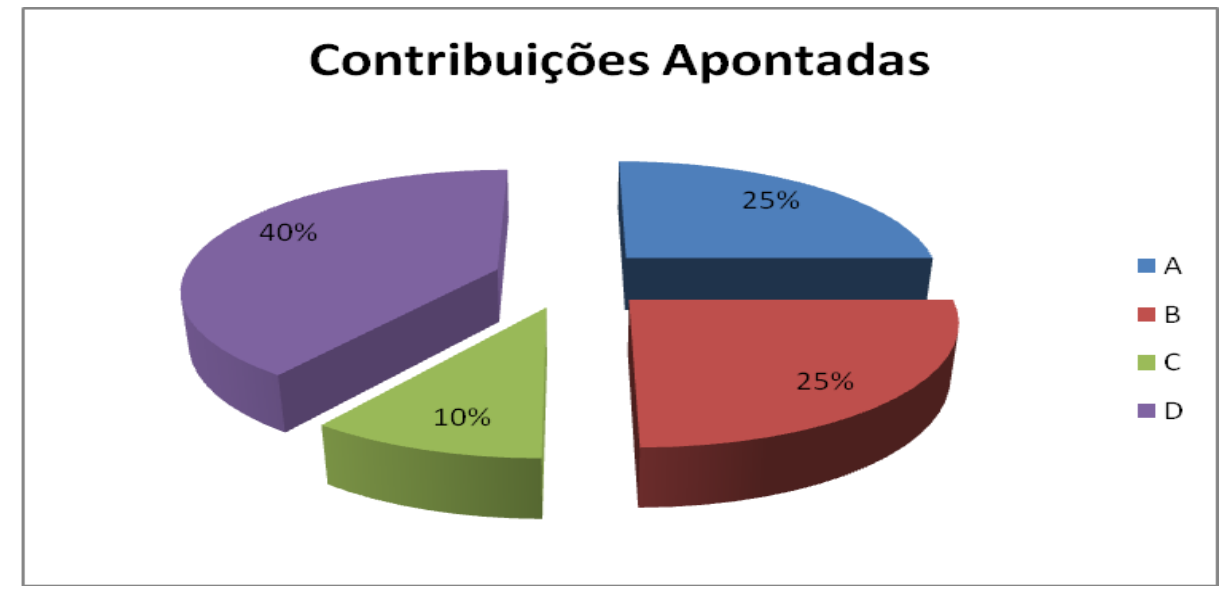

Foi possível verificar que todos os sujeitos envolvidos indicaram alguma "contribuição" promovida pelo software Geogebra, onde 40\% apontaram que além de outras contribuições, o mesmo é capaz de facilitar e estimular a aprendizagem. Referindo-se ao software Geogebra, Gerônimo et. al (2010, p. 11) afirmam que "uma das vantagens do uso deste software é que as construções são dinâmicas, isto é, podem ser modificadas sem a perda dos vínculos geométricos", isso, por consequência permite que o usuário, no caso o aluno, realize uma grande quantidade de experimentações de modo a facilitar a aprendizagem não apenas das proposições geométricas, mas algébricas também.

A última questão preocupou-se em analisar a opinião dos alunos com relação às duas abordagens realizada pelo professor a respeito da Função Polinomial do Primeiro Grau - " $a$ maneira convencional" ou "utilização do software", qual seria a mais significativa para o aprendizado? Praticamente todos os envolvidos afirmaram que a abordagem por meio do software foi a "mais legal", "mais fácil", "preferem usar o computador", respostas estas que vem reforçar a importância de se utilizar recursos que são capazes de tornar a aprendizagem de determinados conceitos mais significativos, como neste caso, foi a utilização de um software. Neste sentido, Marques e Caetano (2002) salientam que muitos programas em multimídia, como é o caso dos softwares, trabalham principalmente mecanismos de percepção, o que permite trazer para a tela do computador uma gama considerável de estímulos de diversos tipos, tais como sons, imagens, textos em movimentos, desenhos, gráficos, dentre outros, "deixando de lado as tarefas que exigem do aluno o exercício de processos cognitivos mais complexos" (MARQUES; CAETANO, 2002, p. 153).

Deste modo, percebe-se que a utilização de recursos tecnológicos, como descrito na intervenção implementada, o software Geogebra se mostrou como uma ferramenta capaz de 
contribuir para que o conteúdo Matemático - Função Polinomial do Primeiro Grau - se tornasse mais dinâmico e significativo, algo que pode favorecer a construção de conhecimentos de conceitos durante a abordagem pedagógica do professor.

\section{CONSIDERAÇÕES FINAIS}

No decorrer da implementação desta proposta foi possível observar o quão significativo foi o envolvimento dos alunos bem como, as discussões promovidas durante a aplicação das atividades, que por sua vez, foram planejadas obedecendo, progressivamente, o grau de dificuldade contido em cada atividade, a fim de exigir do aluno, a compreensão de cada conceito incorporado.

Neste sentido, foi possível perceber a importância da forma como foi elaborada a sequência de atividades, da utilização de material manipulável, da realização das tarefas individuais e em pequenos grupos, das questões levantadas pelo professor, cujo intuito foi de levar o aluno a construir o conceito de Função Polinomial do Primeiro Grau, bem como, sua representação por meio de leis, tabelas e gráficos, e por fim, o uso do software Geogebra, que possibilitou a articulação das construções geométricas, algébricas, tabelas, gráficos e cálculos, de modo a evidenciar a construção de significado deste conteúdo, tendo o aluno como protagonista.

Deste modo, constatou-se que o software Geogebra é um recurso que pode contribuir significativamente para a ação pedagógica do professor de Matemática, evidenciando, com isso, um espaço de discussão para a ampliação da inserção de tecnologias no âmbito escolar, nas mais variadas disciplinas, assim como também, em todos os níveis de ensino.

\section{REFERENCIAIS}

BOGDAN, Robert; BIKLEN, Sari. Investigação Qualitativa em Educação: uma Introdução à Teoria e aos Métodos. Porto: Porto Editora. 1994.

COLL, César; MONEREO, Carles. O impacto das TIC sobre a educação e a psicologia da educação. In: COLL, C., MONEREO, C. e colaboradores. Psicologia da Educação Virtual - aprender e ensinar com as tecnologias da educação e da comunicação. Porto Alegre: Artmed. cap. 1, p. 15-46, 2010.

CRESWELL, John. W. Projeto de Pesquisa: Métodos qualitativo, quantitativo e misto. Porto Alegre. Editora: Artmed. 2a Edição. 2007.

EIVAZIAN, Ana Maria Batista. Uso educacional de aplicativos. In: COX, Kenia Kodel. Informática na educação escolar. Campinas: Autores Associados, 2008.

GERÔNIMO, João Roberto; BARROS, Rui Marcos de Oliveira; FRANCO, ValdeniSoliani. Geometria Euclidiana Plana: um estudo com o software Geogebra. Maringá: Eduem, 2010. 
MARQUES, Adriana Cavalcanti; CAETANO, Josineide da Silva. Utilização da Informática na Escola. In: MERCADO, Luiz Paulo Leopoldo (Org.). Novas tecnologias na educação: reflexões sobre a prática. São Paulo: Edufal, 2002,cap. 4, p. 131-168.

MORAN, José Manuel;MASETTO, Marcos T.;BEHRENS, Marilda Aparecida. Novas Tecnologias e mediação pedagógica. 15. ed. Campinas: Papirus, 2000.

PRENSKY, Marc. Digital Natives, Digital Immigrants.On the Horizon.MCB University Press, vol. 9,n. 5, Oct. 2001. Disponível em:< http://www.marcprensky.com/writing/ Prensky\%20\%20Digital\%20Natives,\%20Digital\%20Immigrants\%20-\%20Part1.pdf>. Acesso em: 02 jun. 2014.

TAJRA, Sanmya Feitosa. Informática na educação: professor na atualidade. São Paulo: Érica, 1998.

TOLEDO, Priscilla Bassitt Ferreira; ALBUQUERQUE, Rosa Almeida Freitas; MAGALHÃES, Àvilo Roberto de. $\mathbf{O}$ comportamento da geração $\mathbf{Z}$ e a influência nas atitudes dos professores. IXSEGeT - Simpósio de excelência em gestão e tecnologia: gestão, inovação e tecnologia para a sustentabilidade. Resende, RJ, out. 2012. Disponível em: <http://www.aedb.br/seget/artigos12/38516548.pdf>. Acesso em: 02 jun. 2014. 